Erschienen in: Jahrbuch der berufs- und wirtschaftspädagogischen Forschung 2018 / Wittmann, Eveline; Frommberger, Dietmar; Ziegler, Birgit (Hrsg.). - Opladen : Verlag Barbara Budrich, 2018. - S. 137-148. - ISBN 978-3-8474-2223-5

https://dx.doi.org/10.3224/84742223

\title{
Bildungshintergrund und Bildungspläne geflüchteter Jugendlicher im System der beruflichen Bildung
}

\author{
Elisabeth Maué, Stephan Schumann und Claudia Diehl
}

\section{Ausgangslage}

Insbesondere in den Jahren 2015 und 2016 sind viele Schutz- und Asylsuchende nach Deutschland gekommen (2015: 890.000; 2016: 280.000; Bundesministerium des Inneren 2017). Deren zügige Eingliederung stellt ein zentrales gesellschaftspolitisches Anliegen dar - sowohl zur Erhöhung der Akzeptanz für Zuwanderung und Pluralisierung als auch zur Vermeidung der Entstehung einer ethnischen Unterschicht mit Folgeproblemen (Koopmanns 2015; Salikutluk, Giesecke \& Kroh 2016). Insofern kommt der strukturellen Integration in Schule, Ausbildung und Beruf eine wesentliche Bedeutung für die Teilhabe am gesellschaftlichen Leben in Deutschland zu (Esser 2001). Eine Herausforderung für die Integration in das Bildungssystem und den Arbeitsmarkt besteht darin, dass die geflüchteten Jugendlichen und Erwachsenen in substanziell von Deutschland verschiedenen Gesellschafts- und Bildungssystemen sozialisiert wurden.

Vor dem Hintergrund, dass die außereuropäische Zuwanderung häufig aus Ländern erfolgt, deren durchschnittliches Bildungsniveau deutlich unter demjenigen Westeuropas liegt sowie angesichts der Altersstruktur der Geflüchteten $-30 \%$ sind 16 bis 25 Jahre alt (Bundesamt für Migration und Flüchtlinge 2017, 22) - ist vielen der Zugang zu tertiärer Bildung (zunächst) verschlossen. Somit spielt das Berufsbildungssystem für die schulische Integration eine entscheidende Rolle. Erstens steht es Personen mit geringer Vorbildung offen und ermöglicht ihnen den Erwerb eines allgemeinbildenden Schulabschlusses, zweitens stellt es eine respektable Alternative zum tertiären Bildungssystem dar und drittens ist es insofern aufnahmefähig, weil derzeit viele Ausbildungsplätze unbesetzt bleiben und vollzeitschulische Alternativen existieren.

In Deutschland haben die Bundesländer auf den hohen Anteil von Asylsuchenden und Geflüchteten, die der Berufsschulpflicht ${ }^{1}$ unterliegen, mit der Einrichtung von spezifischen ein- oder zweijährigen Bildungsgängen reagiert,

1 Die Bundesländer unterscheiden sich dahingehend, ob die speziell für geflüchtete Jugendliche eingerichteten Bildungsgänge und Maßnahmen lediglich berufsschulpflichtigen Jugendlichen offenstehen oder ob auch junge Menschen bis 21 Jahre bzw. teilweise bis 27 Jahre teilnehmen dürfen (Baethge \& Seeber 2016, 31). 
die strukturell der Berufsvorbereitung zugerechnet werden können (Baethge \& Seeber 2016). Jenseits diverser Ausgestaltungsunterschiede ist eine intensive Sprachförderung ein Hauptanliegen dieser zumeist vollzeitschulischen Angebote, da die Förderung der Fähigkeiten in Deutsch eine wesentliche Säule der Integration darstellt (Esser 2001). Die Bereitstellung des Angebots geschieht derzeit jedoch naturgemäß weitgehend ohne eine entsprechende Informationsgrundlage bezüglich der Merkmale der jungen Geflüchteten.

Dem Informationsdefizit begegnet das interdisziplinäre Projekt „Refugees and their early Integration in Society and Education (RISE)“", das eine bildungswissenschaftliche und soziologische Perspektive verbindet. Es geht Fragen nach Merkmalen und Ausgangsvoraussetzungen berufsschulpflichtiger Geflüchteter in Baden-Württemberg sowie deren kurz- und mittelfristiger Integration in das (Aus-)Bildungssystem, das Beschäftigungssystem und die Gesellschaft nach. Zudem wird untersucht, ob sich Merkmale der Integration über die Zeit verändern und welche Faktoren interindividuelle und gruppenspezifische Unterschiede in Integrationsverläufen und Bildungsübergängen erklären.

Dieser Beitrag stellt erste deskriptive Befunde zur schulischen (Vor-)Bildung der geflüchteten Jugendlichen im Herkunftsland und in Deutschland sowie deren Pläne für die nähere schulische bzw. berufliche Zukunft vor.

\section{Bedingungen von Integration}

Mit Bezug zu Esser (2001, 25 ff.) lassen sich auf verschiedenen Ebenen Bedingungsfaktoren für die Sozialintegration von Personen mit Migrations- und Fluchthintergrund unterscheiden. Auf Seiten des Aufnahmelandes spielen die Bedingungen des Arbeits- und Wohnungsmarktes sowie die Gestaltung des Bildungssystems ebenso eine Rolle wie gesellschaftliche Offenheit, Toleranz, soziale und kulturelle Distanzen. Demgegenüber beeinflussen die Größe und institutionelle Vollständigkeit der ethnischen Gemeinde sowie Verwandtschaftsnetzwerke die Aufrechterhaltung der emotionalen Bindung an das Herkunftsland. Bei den Individuen ist für deren Sozialintegration insbesondere das Human-Kapital in Form von Bildungsabschlüssen von Bedeutung. Am wichtigsten jedoch sind Sprachkenntnisse, auch um im Bildungssystem aufnahmelandspezifische Qualifikationen erwerben zu können. Der Aufbau bzw. die Verbesserung von Sprachkenntnissen werden ihrerseits durch interethnische soziale Kontakte gefördert. Gelegenheiten dazu bieten sich insbesondere im Bildungssystem, etwa wenn bei segregierter Beschulung Deutsch als lingua franca im Klassenzimmer fungiert oder bei integrierter Beschulung durch Kontakte zu „deutschen“ MitschülerInnen (SVR 2018). Investitionen des Individuums in den Erwerb von Sprachkenntnissen, Bildungsabschlüssen und inte- 
rethnischen Kontakten stehen mit längerfristigen Lebensplanungen - Rückkehr ins Herkunftsland, Migration in ein anderes Land oder Verbleib im Aufnahmeland - in Zusammenhang (Chiswick \& Miller 2015).

Mit dem Fokus auf die Bildungsbiografie geflüchteter Jugendlicher im Herkunftsland und in Deutschland setzt der Beitrag auf Individualebene bei den Ausgangsvoraussetzungen und den Planungen für ihre nähere Zukunft an.

\section{Junge Geflüchtete in der Berufsvorbereitung in Baden- Württemberg}

In Deutschland unterscheiden sich die Bildungsangebote für geflüchtete Kinder und Jugendliche je nach Bundesland im allgemeinbildenden Bereich (SVR 2018) wie in der beruflichen Bildung (Baethge \& Seeber 2016). Da die Datengrundlage dieses Beitrages einer Studie in beruflichen Schulen in Baden-Württemberg entstammt (Kapitel 5), wird das dortige System näher vorgestellt. In Baden-Württemberg besuchen jugendliche Geflüchtete im berufsschulpflichtigen und berufsschulberechtigten Alter an den beruflichen Schulen sogenannte VABO-Klassen (,Vorqualifizierungsjahr Arbeit/Beruf mit Schwerpunkt Erwerb von Deutschkenntnissen“). Diese verfolgen die Ziele Ankommen in der Schule in Deutschland, Ausbildung von Sprachkompetenz zur Alltagsbewältigung sowie schulische und berufliche Orientierung (Ministerium für Kultus, Jugend und Sport des Landes Baden-Württemberg 2016, 5). Hauptgegenstand ist die Vermittlung von Deutschkenntnissen mit dem Ziel des Sprachniveaus A2 des Gemeinsamen Europäischen Referenzrahmens für Sprachen (GER). Bei Nicht-Erreichen des Sprachniveaus A2 ist eine Wiederholung des VABO vorgesehen. Ein freiwilliger Verbleib im VABO ist möglich, um das Sprachniveau B1 oder B2 zu erlangen. Der Besuch des VABO trägt weder zur Erfüllung der Berufsschulpflicht noch zum Erwerb eines allgemeinbildenden Schulabschlusses bei. Bildungspolitisch intendiert ist im Anschluss an das VABO der Übergang in eine berufliche Ausbildung oder in einen Regelbildungsgang, insbesondere in die Berufsvorbereitungsmaßnahme VAB („Vorqualifizierungsjahr Arbeit/Beruf"), welche zum Erwerb eines Hauptschulabschlusses führt. Ein Wechsel vom VABO in Regelbildungsgänge ist während des Schuljahres möglich, ebenso eine stundenweise Integration in Regelklassen der beruflichen Schule in ausgewählten Fächern (ebd., 13 ff.).

Die berufliche Orientierung im VABO folgt den Phasen Information Praktikum - Bewerbung. Aufgrund des zumeist in den Herkunftsländern der geflüchteten Jugendlichen nicht bekannten Systems der beruflichen Bildung sowie der dortigen zumeist geringen Reputation beruflicher Bildung kommt der Phase der Information eine besondere Bedeutung zu (ebd., 19). 


\section{Erkenntnisstand zur Bildungsbiografie Geflüchteter}

\subsection{Schulische Bildung}

Gemäß Ergebnissen der IAB-BAMF-SOEP-Befragung unter volljährigen Geflüchteten hat ein großer Anteil im Herkunftsland die Mittelschule (33\%) oder eine weiterführende Schule (40 \%) besucht. Die Abschlussquoten fallen etwas geringer aus. Im Durchschnitt sind die Befragten zehn Jahre in die Schule gegangen. Die Schulbesuchsdauer derjenigen mit einem Schulabschluss liegt bei elf Jahren (Brenzel \& Kosyakova 2017, 20). In eine ähnliche Richtung weisen Befunde einer Befragung in bayerischen Berufsschulklassen für neu zugewanderte SchülerInnen, nach der über die Hälfte acht bis zwölf Jahre eine Schule im Herkunftsland besucht hat (Baumann \& Riedl 2016, 90). Die BA/BIBBMigrationsstudie zeigt, dass Geflüchtete mit einem ausländischen Schulabschluss deutlich höher qualifiziert sind als Geflüchtete, die einen Schulabschluss in Deutschland erworben haben (Eberhard, Matthes \& Gei 2017, 446 ff.).

Es zeigen sich große Differenzen je nach Herkunftsland, nicht nur in Bezug auf die durchschnittliche Schulbesuchsdauer, sondern auch hinsichtlich der Streuung innerhalb eines Landes (Baumann \& Riedl 2016, 96 f.; Granato 2017, 27 f.).

\subsection{Berufliche Bildung}

Lediglich jede/r zehnte Geflüchtete absolvierte eine betriebliche Ausbildung (mit Abschluss $8 \%$ ). Ihnen stehen $71 \%$ ohne berufliche Ausbildung gegenüber. $18 \%$ geben ein Studium bzw. eine Promotion an (Abschluss: $12 \%$ ) (Brenzel \& Kosyakova 2017, 21). Demnach spielt eine berufliche Ausbildung in den meisten Herkunftsländern keine oder nur eine untergeordnete Rolle. Berufliche Kenntnisse und Fähigkeiten werden in informellen Kontexten erworben. In diese Richtung weist der Befund, dass $42 \%$ der neu zugewanderten Jugendlichen über Arbeitserfahrungen in ihrem Herkunftsland verfügen, oft in Form von Hilfsarbeiten oder ungelernten Tätigkeiten (Baumann \& Riedl 2016, 102f.; auch Eberhard et al. 2017, 450). Ein Drittel der Geflüchteten der BA/BIBB-Migrationsstudie befand sich in einer beruflichen Ausbildung, 23\% in einer teilqualifizierenden Maßnahme. Zehn Prozent arbeiteten (Eberhard et al. 2017, 448 ff.). 


\section{Forschungsprojekt „Refugees and their early Integration in Society and Education (RISE)“}

Mit der Studie RISE ${ }^{2}$ sollen Einblicke in Bedingungsfaktoren der Sozialintegration der geflüchteten Jugendlichen auf den verschiedenen Ebenen - Individuum, Aufnahmeland, Herkunftsland (Esser 2001) - und deren Interrelation gewonnen werden. Spezifischer geht es im Projekt um die Deskription sowohl von Merkmalen und Ausgangsvoraussetzungen der geflüchteten Jugendlichen, etwa schulische und berufliche Bildungsbiografie im Herkunftsland und in Deutschland und eine umfassende Erhebung der deutschen Sprachkenntnisse (standardisierter Test und Selbsteinschätzung), als auch von Übergängen im Anschluss an die VABO-Klasse. Bedingungen des Aufnahmelandes spiegeln sich beispielsweise in der Wohnsituation oder in der von den SchülerInnen wahrgenommenen Offenheit der deutschen Gesellschaft Geflüchteten gegenüber und in innerethnischen wie interethnischen Kontakten und Netzwerken wider. Fragen zur Lebens- und Zukunftsplanung zielen beispielhaft auf eine Erklärung von Unterschieden in den Bildungs- und Integrationsverläufen ab. Darüber hinaus ist die Frage, ob sich mit zunehmender Dauer des Aufenthalts in Deutschland Einstellungen und Pläne verändern, von Bedeutung.

Der vorliegende Beitrag knüpft mit dem Fokus auf die Bildungsbiografie an die Deskription von Merkmalen und Ausgangsvoraussetzungen der geflüchteten SchülerInnen an. Denn auch wenn mittlerweile einige Befunde zur schulischen und beruflichen Bildung Geflüchteter und Neuzugewanderter vorliegen (Kapitel 4), ist dennoch unklar, inwiefern diese auf die spezielle Gruppe der jugendlichen Geflüchteten übertragbar sind. Insofern geht dieser Beitrag der Frage nach, über welche schulische Bildung geflüchtete Jugendliche in ihrem Herkunftsland verfügen, welche schulische Bildung sie in Deutschland erfahren und welche Pläne sie diesbezüglich haben.

\subsection{Instrumente}

Zum ersten Messzeitpunkt wurden ein Test zur Erfassung des Sprachstands Deutsch, ein Fragebogen sowie ein Kurztest der kognitiven Grundfähigkeit eingesetzt. Die Erhebung wurde online durchgeführt und war in den Sprachen Deutsch, Arabisch, Englisch, Französisch, Paschto, Persisch und Tigrinisch verfügbar (Ausnahme Deutschtest). Die Untersuchungsdurchführung erfolgte durch TestleiterInnen der Universität Konstanz im jeweiligen Klassenverband im PC-Pool der Schulen. Die Befragungszeit umfasste drei Schulstunden.

2 Das Forschungsprojekt RISE wird über das Netzwerk Bildungsforschung der BadenWürttemberg-Stiftung finanziert und vom Regierungspräsidium Freiburg unterstützt. 
Der Deutschtest adressiert basales und auf Alltagssituationen ausgerichtetes Begriffs- und Grammatikwissen sowie Leseverstehen. Der Test wurde auf Grundlage existierender Einstufungstests des Niveaus A1 (GER) entwickelt und im Vorfeld anhand einer vergleichbaren Zielgruppe pilotiert (EAP-Reliabilität $=0.93$ ). Psychometrisch auffällige Items wurden anschließend modifiziert oder eliminiert. Auch zum ersten Messzeitpunkt ist die psychometrische Güte des Tests sehr gut (EAP-Reliabilität $=0.89$; exzellente Itemfits). Nach einer gemeinsamen Instruktions- und Übungsphase standen den Jugendlichen für die 30 Aufgaben 15 Minuten Zeit zur Verfügung.

Der Fragebogen des ersten Messzeitpunktes beinhaltet u. a. die Bereiche Migrationsbiografie, Bildungsbiografie, Bildungsaspirationen, Bildungssystemwissen, Persönlichkeitsmerkmale, Kontakte und Netzwerke, Erfahrungen in und Einstellungen zu Deutschland sowie Zukunftspläne, Sorgen und Zufriedenheit. Eine umfassende Selbsteinschätzung der Deutschkenntnisse und Angaben zum Sprachgebrauch ergänzen die testbasierte Messung des Sprachstandes. Der Fragebogen des zweiten Messzeitpunktes hat vorrangig zum Ziel, Veränderungen und Entwicklungen zwischen den beiden Zeitpunkten nachzuzeichnen.

Die kognitive Grundfähigkeit wurde mit den Subtests „Series“ des CFT20 (Weiß 1980) erfasst. Nach einer gemeinsamen Instruktions- und Übungsphase hatten die SchülerInnen für die 24 Aufgaben sieben Minuten Zeit.

Der Beitrag beantwortet ausgewählte Fragen zur schulischen Bildungsbiografie der geflüchteten Jugendlichen. Diese umfassen die Dauer des Schulbesuchs im Herkunftsland und in Deutschland (in Jahren). Mit Blick auf die näheren schulischen und beruflichen Pläne wurden zum einen die schulischen Bildungsaspirationen über den anvisierten Erwerb eines Schulabschlusses in Deutschland erfasst. Die Antwortkategorien decken konkrete Vorstellungen (Hauptschulabschluss, Mittlerer Bildungsabschluss, Fachabitur, Abitur) und Unwissenheit (Ich weiß es nicht, weil ich das Bildungssystem noch nicht so gut kenne. / Ich habe noch keine Pläne.) ab (Mehrfachnennung möglich). Zum anderen wurde der geplante Übergang im Anschluss an die VABO-Klasse erfragt: Maßnahmen des Übergangssystems (VABO, VAB, Berufseinstiegsjahr), Berufsausbildung, Studium, Austritte aus dem (Berufs-)Bildungssystem (Ich möchte die Schule verlassen und arbeiten. / Ich möchte die Schule verlassen und etwas anderes tun.) sowie Unwissenheit (Ich weiß es nicht, weil ich das Bildungssystem noch nicht so gut kenne. / Ich habe noch keine Pläne.).

\subsection{Datengrundlage}

Das Forschungsdesign umfasst eine Primärdatenerhebung mit zwei Messzeitpunkten. Der erste Messzeitpunkt lag gegen Ende des Schuljahres 2016/2017; der zweite Messzeitpunkt ist für März bis Juli 2018 geplant. 
Die Grundgesamtheit der Studie bilden Geflüchtete in VABO-Klassen im Regierungsbezirk Freiburg. Im Frühling 2017 befanden sich laut Angaben des Regierungspräsidiums Freiburg 2183 SchülerInnen in 131 Klassen in 52 Schulen. Mit einer Ausnahme wurden alle Schulen angeschrieben, von denen in 22 Schulen eine Datenerhebung stattfand. Insgesamt konnten 635 Jugendliche in 60 Klassen befragt werden. Die Geschlechterverteilung entspricht mit $21 \%$ jungen Frauen und 79 \% jungen Männern den Befunden anderer Studien sowie der amtlichen Statistik (Baumann \& Riedl 2016, 56f.; Bundesamt für Migration und Flüchtlinge 2017, 22). Im Durchschnitt sind die Geflüchteten 18.9 Jahre alt $(\mathrm{SD}=2.58 ; \mathrm{n}=605)$, mit einer Spanne von 15 bis $37 \mathrm{Jahren}^{3}$. Auf Basis des Geburtslandes ergeben sich folgende häufigste Herkunftsländer.

Tab. 1: Häufigste Herkunftsländer

\begin{tabular}{lcc}
\hline & Häufigkeit & Prozent \\
\hline Syrien & 183 & 29.5 \\
Afghanistan & 147 & 23.7 \\
Irak & 54 & 8.7 \\
Gambia & 51 & 8.2 \\
Eritrea & 34 & 5.5 \\
Anderes Land & 152 & 24.5 \\
Gesamt & 621 & 100.0 \\
\hline
\end{tabular}

Quelle: eigene Darstellung

Über die Hälfte der SchülerInnen stammen aus Syrien und Afghanistan (53.2 \%). Irak, Gambia und Eritrea sind ebenfalls oft genannte Herkunftsländer, jedoch mit einem jeweils deutlich kleineren Anteil (ähnlich amtliche Statistik, aber weniger SchülerInnen aus Balkanländern: Bundesamt für Migration und Flüchtlinge 2017, 20). Die geringen Anteile anderer Geburtsländer sind zusammengefasst. Insgesamt kommen die befragten Jugendlichen aus mehr als 24 verschiedenen Ländern.

3 Als Grundlage dient das Geburtsjahr. Können die Geflüchteten bei der Einreise nach Deutschland ihr Geburtsdatum nicht eindeutig nennen, erhalten sie das ,neue“ Geburtsdatum 01. Januar plus Jahresangabe. Insofern erscheint die Berücksichtigung des Geburtsmonats zur Bestimmung des Alters wenig sinnvoll. 


\section{Befunde zum Schulbesuch geflüchteter Jugendlicher im Herkunftsland und in Deutschland}

Schulbesuch im Herkunftsland

Fast alle Jugendlichen besuchten in ihrem Herkunftsland eine Schule (Tab. 2), durchschnittlich 7.53 Jahre (SD = 3.50). Sechs Prozent der Geflüchteten sind dort nicht zur Schule gegangen. Der Schulbesuch differiert deutlich: Während etwa ein Drittel (32\%) zehn bis 15 Jahre in die Schule gegangen ist und knapp die Hälfte (48 \%) fünf bis neun Jahre, das Niveau der Sekundarstufe I, kommen $14 \%$ maximal auf vier Jahre, die Dauer der Primarstufe in Deutschland.

Tab. 2: Dauer des Schulbesuchs im Herkunftsland

\begin{tabular}{lcc}
\hline Jahre & Häufigkeit & Prozent \\
\hline 0 & 33 & 5.7 \\
1 & 19 & 3.3 \\
2 & 22 & 3.8 \\
3 & 15 & 2.6 \\
4 & 27 & 4.7 \\
5 & 20 & 3.5 \\
6 & 58 & 10.1 \\
7 & 45 & 7.8 \\
8 & 55 & 9.6 \\
9 & 98 & 17.0 \\
10 & 62 & 10.8 \\
11 & 59 & 10.3 \\
12 & 48 & 8.3 \\
13 & 11 & 1.9 \\
14 & 2 & 0.3 \\
15 & 1 & 0.2 \\
Gesamt & 575 & 100.0 \\
\hline
\end{tabular}

Quelle: eigene Darstellung 
Bezüglich der durchschnittlichen Schulbesuchsdauer existieren signifikante Differenzen zwischen den Herkunftsländern: Geflüchtete aus Afghanistan $(\mathrm{MW}=5.56, \mathrm{SD}=3.75, \mathrm{n}=137)$ und Gambia $(\mathrm{MW}=6.35, \mathrm{SD}=4.37, \mathrm{n}=40)$ haben signifikant kürzer eine Schule besucht als Jugendliche aus Syrien (MW $=8.91, \mathrm{SD}=2.32, \mathrm{n}=173)$ und dem Irak $(\mathrm{MW}=9.12, \mathrm{SD}=2.48, \mathrm{n}=51)$. Zwischen der Kategorie anderes Geburtsland $(\mathrm{MW}=7.56, \mathrm{SD}=3.64, \mathrm{n}=141)$ und Afghanistan und Syrien bestehen zudem signifikante Unterschiede.

\section{Schulbesuch in Deutschland}

Zum Zeitpunkt der Befragung befand sich der Großteil der geflüchteten Jugendlichen im ersten Schuljahr $(72 \% ; \mathrm{n}=381)$, ein Viertel im zweiten Schuljahr $(25 \% ; n=130)$. Die übrigen $3 \%(n=18)$ geben drei oder mehr Jahre an.

Einen Schulabschluss in Deutschland erwerben möchten $79 \%$ der Befragten $(n=466)$, weitere $14 \%(n=81)$ können sich dies vielleicht vorstellen. Die übrigen $8 \%(\mathrm{n}=44)$ streben keinen Schulabschluss in Deutschland an bzw. wissen es noch nicht. Am häufigsten wird der Hauptschulabschluss genannt (42\%), gefolgt vom Abitur (22\%) und dem Mittleren Bildungsabschluss (19\%). Das Fachabitur möchten $9 \%$ erwerben (Mehrfachnennungen möglich). Etwa jede/r Fünfte (18\%) gibt an, das Bildungssystem noch nicht ausreichend zu kennen. $11 \%$ haben noch keine Pläne für einen konkreten Schulabschluss. Hinsichtlich des höchsten angestrebten Schulabschlusses in Deutschland verweist der Kruskal-Wallis-Test auf signifikante Länderunterschiede (Kruskal-Wallis $\mathrm{H}=25.72$; $\mathrm{df}=5 ; \mathrm{p}<.001$ ), welche auf signifikant höhere Aspirationen Jugendlicher aus Syrien als aus Afghanistan und anderen Geburtsländern zurückgehen.

\section{Schuljahr 2017/2018}

In Hinblick auf zeitnahe Übergänge wurden die geflüchteten Jugendlichen nach ihren Plänen für das Schuljahr 2017/2018 gefragt (Tab. 3).

Im Schuljahr 2017/2018 möchte knapp ein Viertel eine Berufsausbildung beginnen (24\%). Ein weiteres Viertel (25\%) strebt mit dem Übertritt ins VAB bzw. BEJ oder AVdual einen Bildungsgang an, der den Erwerb eines Schulabschlusses ermöglicht. Eine Wiederholung des VABO können sich 18 \% vorstellen. Zwar hat jede/r fünfte SchülerIn noch keinen Plan oder kennt das Bildungssystem zu wenig um eine Wahl treffen zu können, doch erscheint ihnen das Verlassen des Bildungssystems, etwa um zu arbeiten, kaum eine geeignete Variante. Die direkte Aufnahme eines Studiums nach der VABO-Klasse planen $8 \%$ der Befragten $(n=45)$.

Bei Betrachtung der Jugendlichen, die im Bildungssystem bleiben möchten, zeigen sich lediglich tendenziell signifikante Unterschiede zwischen den 
Herkunftsländern (Kruskal-Wallis $\mathrm{H}=9.46$; $\mathrm{df}=5 ; \mathrm{p}>.05$ ), welche auf signifikant niedrigere Bildungsgänge Jugendlicher aus dem Irak als aus anderen Geburtsländern zurückzuführen sind.

Tab. 3: Geplante Übertritte nach dem Besuch des VABO-Bildungsgangs

\begin{tabular}{|c|c|c|}
\hline & Häufigkeit & Prozent \\
\hline VABO (Wiederholung) & 100 & 17.8 \\
\hline Berufsvorbereitung (VAB/BEJ/AVdual) & 140 & 24.9 \\
\hline Berufsausbildung & 134 & 23.8 \\
\hline Studium & 45 & 8.0 \\
\hline Arbeiten & 12 & 2.1 \\
\hline Noch keine Pläne & 62 & 11.0 \\
\hline Bildungssystem unbekannt & 52 & 9.3 \\
\hline Anderes $^{\mathrm{a}}$ & 17 & 3.0 \\
\hline Gesamt & 562 & 100.0 \\
\hline
\end{tabular}

Quelle: eigene Darstellung; $V A B=$ Vorqualifizierungsjahr Arbeit/Beruf; BEJ = Berufseinstiegsjahr; $A V$ dual = Ausbildungsvorbereitung dual; ${ }^{a}$ Zusammenfassung der Kategorien Gymnasium, 2-jährige Berufsfachschule und Ich möchte die Schule verlassen und etwas anderes tun.

\section{Diskussion und Schlussbetrachtung}

Das System der beruflichen Bildung spielt für die Integration der geflüchteten Jugendlichen eine entscheidende Rolle. Mit dem Fokus auf dem Erwerb von Kenntnissen der deutschen Sprache und den Möglichkeiten, einen Schulabschluss zu erlangen bzw. nachzuholen, leistet es einen wichtigen Beitrag für die weitere (berufliche) Bildung der Jugendlichen. Deren heterogene schulische und berufliche Vorkenntnisse und Erfahrungen stellen Lehrende wie Lernende vor Herausforderungen (Baumann \& Riedl 2016; Brenzel \& Kosyakova 2017; Granato 2017; Kärner et al. 2016). Die unterschiedlichen Voraussetzungen zeigen sich auch in der vorliegenden Studie bei Betrachtung der Dauer des Schulbesuchs im Herkunftsland, welche nach Herkunftsland differieren. Hinzukommt, dass davon auszugehen ist, dass die Jugendlichen durch die Flucht ihren Schulbesuch für eine (längere) Zeit unterbrechen mussten. 
Das System der beruflichen Bildung hat für einen Großteil der geflüchteten Jugendlichen eine hohe Attraktivität, auch wegen der Option, einen Schulabschluss zu erwerben, welche fast alle Befragten sicher oder vielleicht nutzen möchten. Knapp die Hälfte möchte im Anschluss an das VABO entweder in einen Bildungsgang der Berufsvorbereitung oder in eine Berufsausbildung übergehen. Sie verbleiben nicht nur gemeinsam mit denjenigen, die das VABO wiederholen möchten, in den beruflichen Schulen, sondern sehen ihre Zukunft in der beruflichen (Aus-)Bildung. Hingegen erscheint zumindest in kurzfristiger Perspektive der Plan, direkt nach dem VABO ein Studium aufzunehmen, mit Blick auf (bürokratische) Hürden beim Hochschulzugang unrealistisch.

Inwiefern die Pläne realistisch und z. B. mit einem Ausbildungsvertrag oder der Zusage eines Studienplatzes ,abgesichert“ sind, muss an dieser Stelle offen bleiben. Die Daten des zweiten Messzeitpunktes erlauben eine Beschreibung der Übergänge, die sich für die Jugendlichen ergeben haben, sowie eine Abschätzung, ob es sich bei den Plänen zum ersten Erhebungszeitpunkt um realistische oder idealistische Bildungsaspirationen gehandelt hat.

Der Befund, dass etwa $20 \%$ der SchülerInnen zum Ende des Schuljahres keine Pläne für einen direkten Übertritt haben, unter anderem aufgrund mangelnder Kenntnis des Bildungssystems, schließt an Ergebnisse anderer Studien an (z.B. Gei \& Matthes 2017). Zudem verweist er, entsprechend der Ziele des VABO, auf einen pädagogischen Handlungsbedarf bezüglich Information, Beratung und Berufsorientierung von Beginn an.

Einschränkend ist zu bedenken, dass es sich beim Sample um eine positiv selektierte Stichprobe handelt. Zum Zeitpunkt der ersten Datenerhebung gegen Ende des Schuljahres waren nicht immer alle SchülerInnen einer Klasse anwesend. Zudem war die Teilnahme an der Studie freiwillig. Insofern ist zu vermuten, dass (vorrangig) diejenigen mit einer positiven Einstellung dem Lernen und der Schule gegenüber teilgenommen haben.

Nichtsdestotrotz liefern die Ergebnisse relevante Einblicke in die ersten schulischen Verläufe und potentiellen Übergänge der geflüchteten Jugendlichen an beruflichen Schulen in Deutschland.

\section{Literatur}

Baethge, M. \& Seeber, S. (2016). Herausforderungen der Flüchtlingsmigration für die Strukturen beruflicher Bildung in Deutschland. Expertise im Auftrag des Sachverständigenrats deutscher Stiftungen für Integration und Migration für das Jahresgutachten 2017. Göttingen.

Baumann, B. \& Riedl, A. (2016). Neu zugewanderte Jugendliche und junge Erwachsene an Berufsschulen. Ergebnisse einer Befragung zu Sprach- und Bildungsbiografien. Frankfurt u.a.: Peter Lang. 
Brenzel, H. \& Kosyakova, Y. (2017). 2.2 Bildung im Herkunftsland. In H. Brücker, N. Rother \& J. Schupp (Hrsg.), IAB-BAMF-SOEP-Befragung von Geflüchteten 2016: Studiendesign, Feldergebnisse sowie Analysen zu schulischer wie beruflicher Qualifikation, Sprachkenntnissen sowie kognitiven Potenzialen. Politikberatung kompakt 123 (S. 19-21). Berlin: Deutsches Institut für Wirtschaftsforschung.

Bundesamt für Migration und Flüchtlinge (2017). Das Bundesamt in Zahlen 2016. Asyl, Migration und Integration. Nürnberg: Bundesamt für Migration und Flüchtlinge.

Bundesministerium des Inneren (2017). 280.000 Asylsuchende im Jahr 2016. Pressemitteilung 11.01.2017. Verfügbar unter http:/www.bmi.bund.de/SharedDocs/ Pressemitteilungen/DE/2017/01/asylantraege-2016.html [29.11.2017].

Chiswick, B. R. \& Miller, P. W. (Hrsg.) (2015). Handbook on the Economics of International Immigration. Volume 1A. Oxford \& Amsterdam: Elsevier.

Eberhard, V., Matthes, S. \& Gei, J. (2017). C4 Zugang zu beruflicher Ausbildung - BIBBForschungsergebnisse. In Bundesinstitut für Berufsbildung (Hrsg.), Datenreport zum Berufsbildungsbericht 2017. Bonn.

Esser, H. (2001). Integration und ethnische Schichtung. Arbeitspapiere, Nr. 40. Mannheim: Mannheimer Zentrum für Europäische Sozialforschung.

Gei, J. \& Matthes, S. (2017). Geflüchtete auf dem Weg in Ausbildung - Welche Unterstützung wünschen sie sich? BWP, 46(4), 4-5.

Granato, M. (2017). Bildungsaspirationen, Bildungskapital und Qualifizierungsbedarfe (junger) Geflüchteter. In M. Granato \& F. Neises (Hrsg.), Geflüchtete und berufliche Bildung. Wissenschaftliche Diskussionspapiere, Heft 187 (S. 25-34). Bonn: Bundesinstitut für Beurfsbildung.

Kärner, T., Feldmann, A., Heinrichs, K., Neubauer, J. \& Sembill, D. (2016). Herausforderungen bei der Beschulung von Asylsuchenden und Flüchtlingen im Rahmen von BAFKlassen an beruflichen Schulen: Deskriptive Befunde aus einer Interviewstudie. $W \& E$, $68(2), 58-62$.

Koopmans, R. (2015). Religious Fundamentalism and Hostility against Out-groups: A Comparison of Muslims and Christians in Western Europe. Journal of Ethnic and Migration Studies, 41(1), 33-57.

Ministerium für Kultus, Jugend und Sport des Landes Baden-Württemberg (2016). Leitfaden für das VABO. Vorqualifizierungsjahr Arbeit/Beruf mit Schwerpunkt Erwerb von Deutschkenntnissen (VABO). Überarbeitete Auflage ab Schuljahr 2016/2017. Verfügbar unter http://www.km-bw.de/site/pbs-bw-new/get/documents/KULTUS .Dachmandant/KULTUS/KM-Homepage/Fl\%C3\%BCchtlingsintegration/Leitfaden\% 20VABO \%20Fassung\%20Juli\%202016.pdf [29.11.2017].

Salikutluk, Z., Giesecke, J. \& Kroh, M. (2016). Geflüchtete nahmen in Deutschland später eine Erwerbstätigkeit auf als andere MigrantInnen. DIW Wochenbericht 83(35), 749-757.

SVR (Forschungsbereich beim Sachverständigenrat deutscher Stiftungen für Integration und Migration) (2018). Schule als Sackgasse? Jugendliche Flüchtlinge an segregierten Schulen. Berlin.

Weiß, R. H. (1980). CFT 20. Grundintelligenztest Skala 2. Handanweisung für die Durchführung, Auswertung und Interpretation (2. Auflage). Braunschweig: Georg Westermann. 